\title{
Gravitating Monopole-Antimonopole Systems at Large Scalar Coupling
}

\author{
Jutta Kunz, Ulrike Neemann, Yasha Shnir \\ Institut für Physik, Universität Oldenburg, Postfach 2503 \\ D-26111 Oldenburg, Germany
}

August 14, 2018

\begin{abstract}
We discuss static axially symmetric solutions of $S U(2)$ Einstein-Yang-MillsHiggs theory for large scalar coupling $\lambda$. These regular asymptotically flat solutions represent monopole-antimonopole chain and vortex ring solutions, as well as new configurations, present only for larger values of $\lambda$. When gravity is coupled to the Yang-Mills-Higgs system, branches of gravitating solutions emerge from the flat-space solutions, and extend up to critical values of the gravitational coupling constant. For small scalar coupling only two branches of gravitating solutions exist, where the second branch connects to a generalized Bartnik-McKinnon solution. For large scalar coupling, however, a plethora of gravitating branches can be present and indicate the emergence of new flat-space branches.
\end{abstract}

PACS numbers: $\quad 14.80 . \mathrm{Hv}, 11.15 \mathrm{Kc}$ 


\section{Introduction}

The 't Hooft-Polyakov monopole [1] represents but the simplest of a rich variety of regular classical non-perturbative finite energy solutions of $S U(2)$ Yang-Mills-Higgs (YMH) theory. Besides this spherically symmetric solution, axially symmetric multimonopoles [2, 3, 4, 5], monopole-antimonopole pairs (MAPs) [6, 7, 8], monopole-antimonopole chains (MACs) [9], and vortex ring solutions [9, 10] are known, as well as multimonopole solutions with only discrete symmetries [11].

In the Bogomol'nyi-Prasad-Sommerfield (BPS) limit of vanishing Higgs potential, the repulsive and attractive forces between two monopoles exactly compensate for any separation, thus BPS monopoles experience no net interaction [12]. As the scalar field becomes massive, the fine balance of forces between the monopoles is broken, since the attractive Yukawa interaction becomes short-ranged. Consequently non-BPS monopoles experience repulsion [5]. This repulsion can be overcome by the inclusion of gravity, which allows for bound multimonopoles [13].

In monopole and multimonopole solutions, the nodes of the Higgs field are associated with the location of the magnetic charges. This also holds for monopole-antimonopole pair and chain solutions, where $m$ monopoles and antimonopoles are located symmetrically on the symmetry axis in alternating order [6, 7, 8, 9]. When the magnetic charge $n$ of the individual monopoles and antimonopoles becomes large, the balance between the repulsive and attractive interactions of the monopoles and antimonopoles is shifted. For vanishing Higgs potential, the structure of the solutions then changes completely, and the zeros of the Higgs field form one or more rings, centered around the symmetry axis. Thus vortex ring solutions arise [9].

When the scalar coupling $\lambda$ is small, the transition from MAPs and MACs to vortex ring solutions appears for magnetic charge $n=3$ [9. For larger scalar coupling $\lambda$, however, bifurcations arise, where new pairs of solutions appear [10]. Thus MAP or MAC solutions and vortex ring solutions may coexist beyond some critical value of $\lambda$. Moreover new configurations with mixed node structure arise, possessing isolated nodes on the symmetry axis as well as vortex rings [10]. The strength of the Higgs selfinteraction is clearly critical for the existence and the properties of these new equilibrium configurations.

We here address such monopole-antimonopole systems in flat-space, reviewing and supplementing previous results [10]. In particular, we obtain new sets of solutions associated with further bifurcations at larger values of the scalar coupling, both in the topologically trivial and the non-trivial sector. While the mass of these configurations increases with the scalar coupling, it is largely determined by their internal structure. We confirm our previous conjecture, that for large scalar coupling, MAC solutions appear to be the energetically most favourable configurations.

The balance of forces changes again, when gravity is coupled to YMH theory. This 
makes the investigation of self-gravitating MAPs, MACs and vortex ring solutions of Einstein-Yang-Mills-Higgs (EYMH) theory very interesting. Depending on the strength of gravity, the structure of the solutions may change again [14]. Also new solutions may arise, which do not possess a flat-space limit [15, 16, 17]. But gravity cannot become too strong, for such regular equilibrium configurations to exist, since beyond a certain gravitational coupling strength, a horizon is expected to form [15].

For gravitating monopoles and multimonopoles a degenerate horizon forms indeed at a critical value of the gravitational coupling [15, 18, 13]. For monopole-antimonopole systems, however, no horizon is seen to form. Instead the flat-space branch of regular graviting monopoles bifurcates with a second branch at a maximal value of the gravitational coupling. This second branch then extends backwards and, in the limit of vanishing coupling constant $\alpha$, it becomes linked to a generalized Bartnik-McKinnon (BM) solution of Einstein-Yang-Mills (EYM) theory [19, 20, 21].

Anticipating new phenomena [18, 21, we here address the effect of gravity on monopole-antimonopole systems at large values of the scalar coupling. Indeed, as $\lambda$ becomes large, we observe a rich pattern of bifurcating branches of solutions arising at finite gravitational coupling strength, which encompasses all possible combinations of isolated nodes and vortex rings. Moreover, certain bifurcations of the gravitating branches of solutions turn out to be precursors of the bifurcations of the flat-space solutions, and, in the limiting case of infinite $\lambda$, particular bifurcations become linked to the corresponding generalized BM solutions of EYM theory.

In section II we present the action of EYMH theory, the axially symmetric ansatz and the boundary conditions. We then discuss the properties of gravitating monopoleantimonopole paris, chains and vortex rings at large $\lambda$ in section III, focussing on monopole-antimonopole systems with $m=2,3,4$ and $n=3$. We give our conclusions in section IV.

\section{$2 S U(2)$ EYMH Action and Axially Symmetric Ansatz}

\subsection{Action}

We consider the $S U(2)$ Einstein-Yang-Mills-Higgs theory with action

$$
S=\int\left\{\frac{R}{16 \pi G}-\frac{1}{2} \operatorname{Tr}\left(F_{\mu \nu} F^{\mu \nu}\right)-\frac{1}{4} \operatorname{Tr}\left(D_{\mu} \Phi D^{\mu} \Phi\right)-\frac{\lambda}{4} \operatorname{Tr}\left[\left(\Phi^{2}-\eta^{2}\right)^{2}\right]\right\} \sqrt{-g} d^{4} x
$$

with curvature scalar $R, s u(2)$ field strength tensor

$$
F_{\mu \nu}=\partial_{\mu} A_{\nu}-\partial_{\nu} A_{\mu}+i e\left[A_{\mu}, A_{\nu}\right],
$$


gauge potential $A_{\mu}=A_{\mu}^{a} \tau^{a} / 2$, and covariant derivative of the Higgs field $\Phi=\Phi^{a} \tau^{a}$ in the adjoint representation

$$
D_{\mu} \Phi=\partial_{\mu} \Phi+i e\left[A_{\mu}, \Phi\right] .
$$

Here $G$ and $e$ denote the gravitational and gauge coupling constants, respectively, $\eta$ denotes the vacuum expectation value of the Higgs field, and $\lambda$ represents the strength of the scalar coupling.

Under $S U(2)$ gauge transformations $U$, the gauge potentials transform as

$$
A_{\mu}^{\prime}=U A_{\mu} U^{\dagger}+\frac{i}{e}\left(\partial_{\mu} U\right) U^{\dagger}
$$

and the Higgs field transforms as

$$
\Phi^{\prime}=U \Phi U^{\dagger}
$$

The nonzero vacuum expectation value of the Higgs field breaks the non-Abelian $S U(2)$ gauge symmetry to the Abelian U(1) symmetry. The particle spectrum of the theory then consists of a massless photon, two massive vector bosons of mass $M_{v}=e \eta$, and a massive scalar field $M_{s}=\sqrt{2 \lambda} \eta$. In the BPS limit the scalar field also becomes massless, since $\lambda=0$, i.e., the Higgs potential vanishes.

Variation of the action (11) with respect to the metric $g^{\mu \nu}$ leads to the Einstein equations

$$
G_{\mu \nu}=R_{\mu \nu}-\frac{1}{2} g_{\mu \nu} R=8 \pi G T_{\mu \nu}
$$

with stress-energy tensor

$$
\begin{aligned}
T_{\mu \nu} & =g_{\mu \nu} L_{M}-2 \frac{\partial L_{M}}{\partial g^{\mu \nu}} \\
& =2 \operatorname{Tr}\left(F_{\mu \alpha} F_{\nu \beta} g^{\alpha \beta}-\frac{1}{4} g_{\mu \nu} F_{\alpha \beta} F^{\alpha \beta}\right) \\
& +\operatorname{Tr}\left(\frac{1}{2} D_{\mu} \Phi D_{\nu} \Phi-\frac{1}{4} g_{\mu \nu} D_{\alpha} \Phi D^{\alpha} \Phi\right)-\frac{\lambda}{8} g_{\mu \nu} \operatorname{Tr}\left(\Phi^{2}-\eta^{2}\right)^{2} .
\end{aligned}
$$

Variation with respect to the gauge field $A_{\mu}$ and the Higgs field $\Phi$ leads to the matter field equations,

$$
\begin{aligned}
& \frac{1}{\sqrt{-g}} D_{\mu}\left(\sqrt{-g} F^{\mu \nu}\right)-\frac{1}{4} i e\left[\Phi, D^{\nu} \Phi\right]=0, \\
& \frac{1}{\sqrt{-g}} D_{\mu}\left(\sqrt{-g} D^{\mu} \Phi\right)+\lambda\left(\Phi^{2}-\eta^{2}\right) \Phi=0
\end{aligned}
$$

respectively. 


\subsection{Static axially symmetric Ansatz}

To obtain gravitating static axially symmetric solutions, we employ isotropic coordinates [20, 13, 21]. In terms of the spherical coordinates $r, \theta$ and $\varphi$ the isotropic metric reads

$$
d s^{2}=-f d t^{2}+\frac{m}{f} d r^{2}+\frac{m r^{2}}{f} d \theta^{2}+\frac{l r^{2} \sin ^{2} \theta}{f} d \varphi^{2}
$$

where the metric functions $f, m$ and $l$ are functions of the coordinates $r$ and $\theta$, only. The $z$-axis $(\theta=0, \pi)$ represents the symmetry axis. Regularity on this axis requires

$$
\left.m\right|_{\theta=0, \pi}=\left.l\right|_{\theta=0, \pi}
$$

We take a purely magnetic gauge field, $A_{0}=0$, and parametrize the gauge potential and the Higgs field by the Ansatz [9]

$$
\begin{aligned}
A_{\mu} d x^{\mu} & =\left(\frac{K_{1}}{r} d r+\left(1-K_{2}\right) d \theta\right) \frac{\tau_{\varphi}^{(n)}}{2 e} \\
& -n \sin \theta\left(K_{3} \frac{\tau_{r}^{(n, m)}}{2 e}+K_{4} \frac{\tau_{\theta}^{(n, m)}}{2 e}\right) d \varphi \\
\Phi & =\eta\left(\Phi_{1} \tau_{r}^{(n, m)}+\Phi_{2} \tau_{\theta}^{(n, m)}\right) .
\end{aligned}
$$

where the $s u(2)$ matrices $\tau_{r}^{(n, m)}, \tau_{\theta}^{(n, m)}$, and $\tau_{\varphi}^{(n)}$ are defined as products of the spatial unit vectors

$$
\begin{aligned}
\hat{e}_{r}^{(n, m)} & =(\sin (m \theta) \cos (n \varphi), \sin (m \theta) \sin (n \varphi), \cos (m \theta)) \\
\hat{e}_{\theta}^{(n, m)} & =(\cos (m \theta) \cos (n \varphi), \cos (m \theta) \sin (n \varphi),-\sin (m \theta)) \\
\hat{e}_{\varphi}^{(n)} & =(-\sin (n \varphi), \cos (n \varphi), 0)
\end{aligned}
$$

with the Pauli matrices $\tau^{a}=\left(\tau_{x}, \tau_{y}, \tau_{z}\right)$, i.e.

$$
\begin{aligned}
\tau_{r}^{(n, m)} & =\sin (m \theta) \tau_{\rho}^{(n)}+\cos (m \theta) \tau_{z} \\
\tau_{\theta}^{(n, m)} & =\cos (m \theta) \tau_{\rho}^{(n)}-\sin (m \theta) \tau_{z} \\
\tau_{\varphi}^{(n)} & =-\sin (n \varphi) \tau_{x}+\cos (n \varphi) \tau_{y}
\end{aligned}
$$

with $\tau_{\rho}^{(n)}=\cos (n \varphi) \tau_{x}+\sin (n \varphi) \tau_{y}$. For $m=2, n=1$ the Ansatz corresponds to the one for the monopole-antimonopole pair solutions [7, 8, 16], while for $m=1, n>1$ it corresponds to the Ansatz for axially symmetric multimonopoles [3, 5, 13]. The four gauge field functions $K_{i}$ and two Higgs field functions $\Phi_{i}$ depend on the coordinates $r$ and $\theta$, only. All profile functions are even or odd w.r.t. reflection symmetry, $z \rightarrow-z$. 
The gauge transformation

$$
U=\exp \left\{i \Gamma(r, \theta) \tau_{\varphi}^{(n)} / 2\right\}
$$

leaves the Ansatz form-invariant [22]. To construct regular solutions we have to fix the gauge [5]. Here we impose the gauge condition [9]

$$
r \partial_{r} K_{1}-\partial_{\theta} K_{2}=0 \text {. }
$$

With this Ansatz the equations of motion reduce to a set of 9 coupled partial differential equations, to be solved numerically subject to the set of boundary conditions, discussed below.

\subsection{Boundary conditions}

To obtain globally regular asymptotically flat solutions with the proper symmetries, we must impose appropriate boundary conditions [13, 9].

Boundary conditions at the origin

Regularity of the solutions at the origin $(r=0)$ requires for the metric functions the boundary conditions

$$
\left.\partial_{r} f(r, \theta)\right|_{r=0}=\left.\partial_{r} m(r, \theta)\right|_{r=0}=\left.\partial_{r} l(r, \theta)\right|_{r=0}=0,
$$

whereas the gauge field functions $K_{i}$ satisfy

$$
K_{1}(0, \theta)=K_{3}(0, \theta)=K_{4}(0, \theta)=0, \quad K_{2}(0, \theta)=1,
$$

and the Higgs field functions $\Phi_{i}$ satisfy

$$
\begin{gathered}
\sin (m \theta) \Phi_{1}(0, \theta)+\cos (m \theta) \Phi_{2}(0, \theta)=0, \\
\left.\partial_{r}\left[\cos (m \theta) \Phi_{1}(r, \theta)-\sin (m \theta) \Phi_{2}(r, \theta)\right]\right|_{r=0}=0,
\end{gathered}
$$

i.e. $\Phi_{\rho}(0, \theta)=0, \partial_{r} \Phi_{z}(0, \theta)=0$.

Boundary conditions at infinity

Asymptotic flatness imposes on the metric functions of the solutions at infinity $(r=$ $\infty)$ the boundary conditions

$$
f \longrightarrow 1, \quad m \longrightarrow 1, \quad l \longrightarrow 1 .
$$

Considering the gauge field at infinity, we require that solutions in the vacuum sector $Q=0$, where $m=2 k$, tend to a gauge transformed trivial solution,

$$
\Phi \longrightarrow \eta U \tau_{z} U^{\dagger}, \quad A_{\mu} \longrightarrow \frac{i}{e}\left(\partial_{\mu} U\right) U^{\dagger},
$$


and that solutions in the sector with topological charge $n$, where $m=2 k+1$, tend to

$$
\Phi \longrightarrow U \Phi_{\infty}^{(1, n)} U^{\dagger}, \quad A_{\mu} \longrightarrow U A_{\mu \infty}^{(1, n)} U^{\dagger}+\frac{i}{e}\left(\partial_{\mu} U\right) U^{\dagger}
$$

where

$$
\Phi_{\infty}^{(1, n)}=\eta \tau_{r}^{(1, n)}, \quad A_{\mu \infty}^{(1, n)} d x^{\mu}=\frac{\tau_{\varphi}^{(n)}}{2 e} d \theta-n \sin \theta \frac{\tau_{\theta}^{(1, n)}}{2 e} d \varphi
$$

is the asymptotic solution of a charge $n$ multimonopole, and $U=\exp \left\{-i k \theta \tau_{\varphi}^{(n)}\right\}$, both for even and odd $m$.

In terms of the functions $K_{1}-K_{4}, \Phi_{1}, \Phi_{2}$ these boundary conditions read

$$
\begin{gathered}
K_{1} \longrightarrow 0, \quad K_{2} \longrightarrow 1-m, \\
K_{3} \longrightarrow \frac{\cos \theta-\cos (m \theta)}{\sin \theta} \quad m \text { odd }, \quad K_{3} \longrightarrow \frac{1-\cos (m \theta)}{\sin \theta} m \text { even }, \\
K_{4} \longrightarrow \frac{\sin (m \theta)}{\sin \theta}, \\
\Phi_{1} \longrightarrow 1, \quad \Phi_{2} \longrightarrow 0 .
\end{gathered}
$$

Boundary conditions along the symmetry axis

The boundary conditions along the $z$-axis $(\theta=0$ and $\theta=\pi)$ are determined by the symmetries. The metric functions satisfy along the axis

$$
\partial_{\theta} f=\partial_{\theta} m=\partial_{\theta} l=0,
$$

whereas the matter field functions satisfy

$$
K_{1}=K_{3}=\Phi_{2}=0, \quad \partial_{\theta} K_{2}=\partial_{\theta} K_{4}=\partial_{\theta} \Phi_{1}=0 .
$$

\subsection{Mass and charge}

Let us introduce the dimensionless coordinate $x$ and the dimensionless coupling constant $\alpha[15]$,

$$
x=\frac{e \alpha}{\sqrt{4 \pi G}} r, \quad \alpha=\sqrt{4 \pi G} \eta
$$

The limit $\alpha \rightarrow 0$ can be approached in two different ways: $G \rightarrow 0$ while the Higgs vacuum expectation value $\eta$ remains finite (flat-space limit), and $\eta \rightarrow 0$ while Newton's constant $G$ remains finite. Corresponding to these two limits two branches of solutions may exist.

The dimensionless mass $M$ of the solutions is obtained from the asymptotic expansion of the metric function $f$,

$$
M=\frac{1}{2 \alpha^{2}} \lim _{x \rightarrow \infty} x^{2} \partial_{x} f
$$


The asymptotic expansion of the gauge fields yields the dimensionless magnetic charge $P$ of the solutions,

$$
P=\frac{n}{2}\left[1-(-1)^{m}\right],
$$

i.e., solutions in the topologically trivial sector have no charge, $P=0$, whereas solutions in the non-trivial sectors have charge $P=n$.

\section{$3 \quad$ Numerical Results}

We have constructed numerically regular gravitating monopole-antimonopole systems, including monopole-antimonopole chain and vortex ring solutions. We here focus on solutions with $m=2,3$ and 4 and $n=3$. In particular, we illustrate the plethora of solutions appearing, when the scalar coupling $\lambda$ is sufficiently large. At the same time we study the dependence of the properties of these solutions on the gravitational coupling strength $\alpha$.

We first briefly address the numerical procedure. Then we present our results for monopole-antimonole chains, vortex rings and configurations with more complicated structure, beginning with the $m=2, n=3$ systems.

\subsection{Numerical procedure}

To construct solutions subject to the above boundary conditions, we map the semiinfinite range of the radial coordinate $r$ onto the closed unit interval of the new compactified radial variable $\bar{x} \in[0: 1]$,

$$
\bar{x}=\frac{r}{1+r},
$$

i.e., the partial derivative with respect to the radial coordinate changes according to

$$
\partial_{r} \rightarrow(1-\bar{x})^{2} \partial_{\bar{x}}
$$

The numerical calculations are then performed with help of the package FIDISOL, based on the Newton-Raphson iterative procedure [23]. Technically, the system of non-linear elliptic partial differential equations of second order is first discretized on a non-equidistant grid in $\bar{x}$ and $\theta$. Typical grids used have sizes $60 \times 40$ covering the integration domain $0 \leq \bar{x} \leq 1$ and $0 \leq \theta \leq \pi / 2$. The numerical errors are typically of order of $10^{-4}$.

\subsection{MAPs and vortex rings: $m=2, n=3$}

\subsubsection{Flat space solutions}

Monopole-antimonopole pairs (MAPs) reside in the topologically trivial sector. These solutions represent unstable equilibrium configurations and can be thought of as con- 
sisting of two solitons of individual charges $\pm n$. The position of each constituent can be identified according to the location of the nodes of the Higgs field. The separation between the poles depends on the subtle interplay of forces and thus on the strength of the scalar coupling $\lambda[8]$.

The MAPs with $n=1$ exist for arbitrary values of $\lambda$. They have two isolated nodes on the symmetry axis, indicating the position of the poles, and their energy density possesses two local maxima, associated with the nodes of the Higgs field [16].

As the charge of the poles increases, the interaction between the nonabelian matter fields becomes stronger than in the case of unit charge constituents, leading to new types of solutions. Indeed, for small and vanishing values of $\lambda$, MAPs with $n>2$ no longer exist. Instead solutions of vortex ring type emerge, where the Higgs field vanishes on a ring centred around the symmetry axis in the $x y$ plane [9]. These flat-space vortex ring solutions form the fundamental $\lambda$-branches, which persist for arbitrary values of $\lambda$. As the scalar coupling strength increases, however, bifurcations are observed, where pairs of additional flat-space solutions emerge at critical values of $\lambda[10]$.

For $n=3$, in particular, a pair of MAP solutions arises at the critical value $\lambda_{c}^{1}=$ 1.382. The $\lambda$-dependence of these flat-space solutions is demonstrated in Fig. 1, where we show their mass and the location of the nodes of their Higgs field configurations. At the bifurcation, the mass of the new MAP solutions is higher than the mass of the vortex ring solution. For large values of $\lambda$, however, the MAP solution on the lower mass branch becomes energetically favoured. Note, that the node structure of the solutions on the upper mass branch changes from MAP to vortex ring, as $\lambda$ increases, but the radius of the ring remains small.
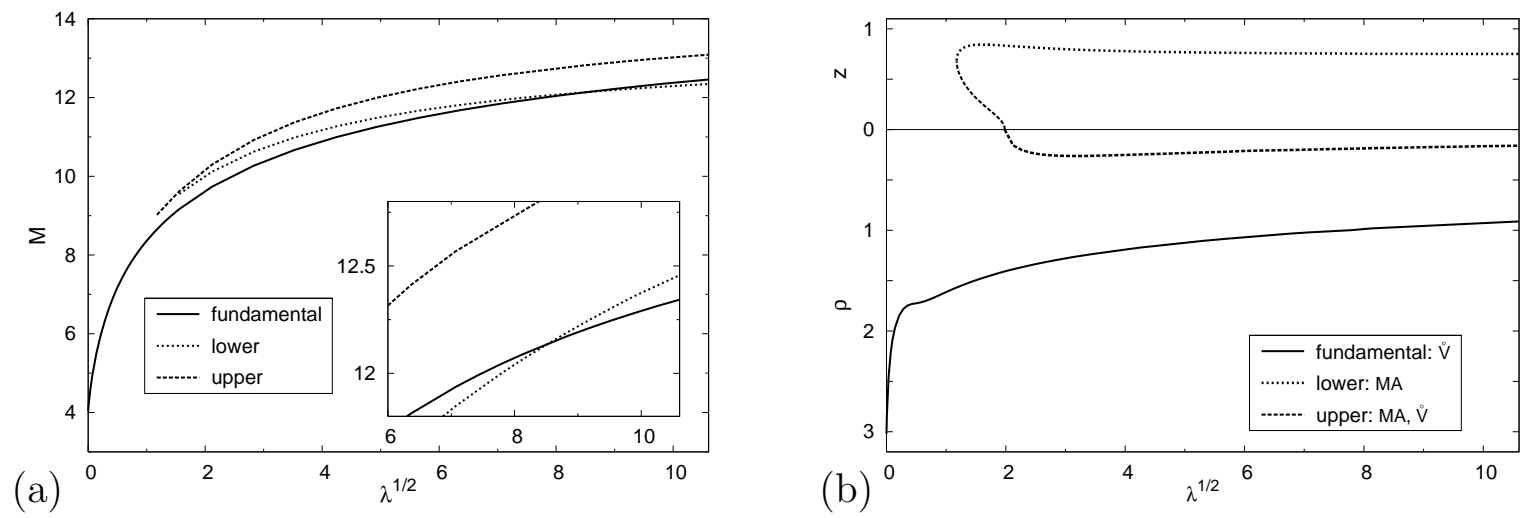

Figure 1: (a) The mass $M$ of the flat-space solutions on the fundamental $\lambda$-branch as well as on the two additional $\lambda$-branches for $m=2, n=3$ solutions. (b) The location of the isolated nodes $z$ and the radius $\rho$ of the vortex ring of the Higgs field for the same set of solutions. (See Table 1 for the notation of the node and vortex ring configurations of the Higgs field.) 


\begin{tabular}{|c|c|c|}
\hline MA & & $\begin{array}{c}\text { A monopole-antimonopole pair with a } \\
\text { monopole above and an antimonopole below } \\
\text { the } x y \text {-plane on the symmetry axis. }\end{array}$ \\
\hline$\dot{V}$ & & A vortex ring in the $x y$-plane. \\
\hline
\end{tabular}

Table 1: Configurations of nodes and vortex rings of the Higgs field for $m=2, n=3$ solutions.

\subsubsection{Gravitating solutions}

When gravity is coupled, branches of gravitating solutions arise from these flat-space configurations. For sufficiently large gravitational coupling $\alpha$, however, a gravitational instability should develop. Therefore regular gravitating solutions will exist only up to a maximal value of $\alpha$.

The critical behaviour of these $\alpha$-branches, emerging from flat-space solutions, is elucidated by numerical investigation. As such an $\alpha$-branch approaches its maximal value of $\alpha$, it merges with a second $\alpha$-branch of regular gravitating solutions, where $\alpha_{\max }$ is the endpoint of both branches. The nature of the second branch, however, depends on the nature of the first branch and on the value of $\lambda$.

\section{Fundamental solutions}

When considering sets of solutions, emerging from the fundamental flat-space $\lambda$ branch, we always find two $\alpha$-branches, where the solutions on the second $\alpha$-branches shrink to zero size in the limit $\alpha \rightarrow 0$, while their mass diverges. By scaling the coordinates and the Higgs field of the solutions via

$$
\hat{x}=\frac{x}{\alpha}, \quad \hat{\Phi}=\alpha \Phi,
$$

one realizes that the second $\alpha$-branches connect to limiting EYM solutions, when $\alpha \rightarrow 0$, solutions with finite scaled size and finite scaled mass $\hat{M}$ [16],

$$
\hat{M}=\alpha M
$$

Indeed, after the scaling, the field equations do not depend on $\alpha$. Instead $\alpha$ appears in the asymptotic boundary conditions of the Higgs field, $|\hat{\Phi}| \rightarrow \alpha$. The Higgs field then becomes trivial on the second $\alpha$-branches as $\alpha \rightarrow 0$, and the solutions thus approach EYM solutions. Depending on $n$, the limiting solutions correspond to the lowest BM or a generalized BM solution, i.e., the solutions on these second $\alpha$-branches possess no flat-space limit. 
The two $\alpha$-branches are illustrated in Fig. 2 for solutions with $m=2, n=3$ at scalar coupling strength $\lambda=0, \lambda=2.0$ and $\lambda=4.5$. The figure exhibits the mass and the scaled mass of these solutions and the location of their nodes. Note, that they always correspond to vortex ring solutions.
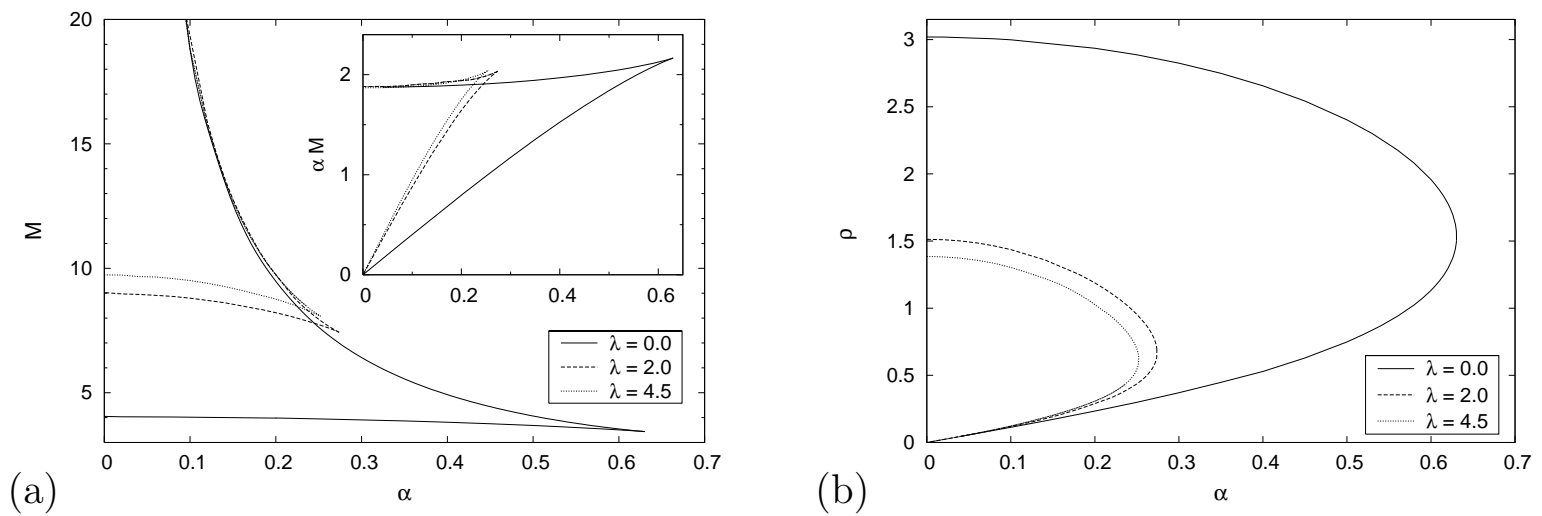

Figure 2: (a) The mass $M$ and the scaled mass $\alpha M$ of $m=2, n=3$ solutions, emerging from the fundamental flat-space $\lambda$-branch versus $\alpha$. (b) The radius $\rho$ of the vortex ring of the Higgs field for the same set of solutions.

New solutions: intermediate $\lambda$

The second $\alpha$-branch always connects to an EYM solution, when the first $\alpha$-branch emerges from a fundamental $\lambda$-branch solution. But for the $\alpha$-branches of the new flatspace solutions, which exist only beyond a critical value of $\lambda$, one may anticipate a different pattern, because these solutions always come in pairs.

In the presence of gravity, each of the solutions of such a pair forms an $\alpha$-branch, extending up to a maximal value of $\alpha$. But the critical behaviour at $\alpha_{\text {max }}$ now depends on the strength of the scalar coupling $\lambda$. In the simplest case, observed for not too large values of $\lambda$, the two $\alpha$-branches simply merge and end at $\alpha_{\max }$. Indeed, this critical behaviour is different from the above, since both branches merging at $\alpha_{\max }$ have flatspace limits.

This is illustrated in Fig. 3, where we exhibit the mass and the location of the nodes for $m=2, n=3$ configurations at $\lambda=2.0, \lambda=2.645, \lambda=3.645, \lambda=4.5, \lambda=5.25$ and $\lambda=12.5$ versus the coupling constant $\alpha$. Evidently, for the smaller $\lambda$ values, a first $\alpha$-branch of solutions originating from the lower mass flat-space solution, extends up to a maximal value $\alpha_{\max }$, where it merges with a second $\alpha$-branch, originating from the upper mass flat-space solution. The solutions on these branches correspond to MAPs.

However, as $\lambda$ increases, a new phenomenon arises on the second $\alpha$-branch, as it evolves back from $\alpha_{\max }$ towards the flat-space solution. As seen in Fig. 3, at a critical value of $\lambda(\lambda \approx 4.5)$ a bifurcation appears on the second $\alpha$-branch, giving rise to two additional $\alpha$-branches of solutions. 

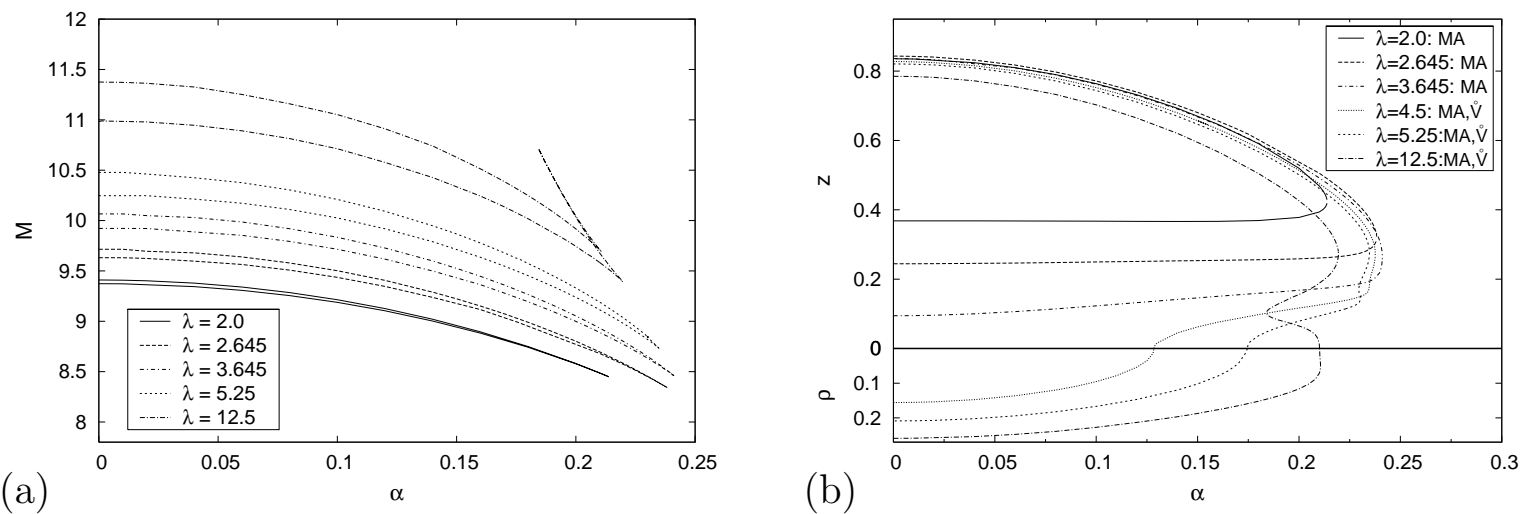

Figure 3: (a) The mass $M$ of $m=2, n=3$ solutions, related to the new flat-space $\lambda$-branches versus $\alpha$ for several values of the scalar coupling $\lambda$. (b) The location of the isolated nodes $z$ and radius $\rho$ of the vortex ring of the Higgs field for the same set of solutions. (See Table 1 for the notation of the node and vortex ring configurations of the Higgs field.)

These 3rd and 4th $\alpha$-branches exist beyond a minimal value of $\alpha, \alpha_{\min }$, and below a respective maximal value of $\alpha$, where they merge with the first and second $\alpha$-branch, respectively. The range $\alpha_{\min } \leq \alpha_{\max }^{(i)}$, where these additional branches exist, increases with increasing $\lambda$. In Fig. 3 the additional branches manifest in the mass of the $\lambda=12.5$ solutions as a higher mass spike. Note, that in this range of $\lambda$ vortex ring solutions arise on a part of the second $\alpha$-branch.

The emergence and evolution of these new $\alpha$-branches is illustrated further in Fig. 4, where we exhibit the values of the metric functions $f$ and $l$ at the origin, $f(0)$ and $l(0)$. In particular, $l(0)$ clearly exhibits the various branches and critical points and therefore represents an instructive means to clarify the pattern of solutions.

To complement the previous discussion of the new solutions, let us consider the $\lambda$ dependence of the solutions for fixed values of $\alpha$. In Fig. 5 we exhibit the mass, the location of the nodes, and the values of the metric functions at the origin versus $\lambda$ for $m=2, n=3$ configurations at $\alpha=0.2, \alpha=0.21, \alpha=0.22, \alpha=0.23$ and $\alpha=0.24$. One clearly recognizes the new solutions, present in a certain region of parameter space.

Since both the scalar interaction and gravity are attractive, one can expect a similar effect concerning the changing structure of the nodes, when $\lambda$ is increased at fixed $\alpha$ as when $\alpha$ is increased at fixed $\lambda$. For the new flat-space upper mass $\lambda$-branch, a transition from MAPs to vortex rings appears at $\lambda=3.941$ [10].

As the gravitational coupling constant $\alpha$ increases, the pattern of the nodes of the solutions changes significantly as well, and there are no solutions with vortex rings (except for those connected to the fundamental $\lambda$-branch), when $\alpha>0.215$ for any value of $\lambda$. This pattern is illustrated in Figure 5, where the location of the nodes, both isolated zeros and vortex rings, is shown versus the scalar coupling for several values of 


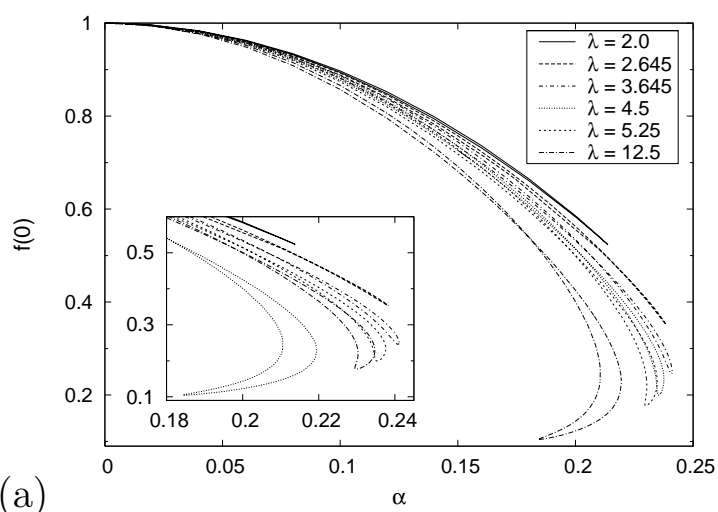

(a)

Figure 4: The values of metric functions at the origin, $f(0)$ (a) and $l(0)$ (b) of $m=2, n=3$ solutions, related to the new flat-space $\lambda$-branches versus $\alpha$ for several intermediate values of the scalar coupling $\lambda$.

$\alpha$. Evidently, the coupling to gravity restricts the range of possible values of $\lambda$, where these solutions can exist, and this restriction is the stronger, the larger $\alpha$, shrinking the domain of existence to zero at a critical value of $\alpha$.

New solutions: very large $\lambda$

As $\lambda$ increases further, the new $\alpha$-branches extend further backwards. Along these branches the mass of the solutions increases further as $\alpha$ decreases. At the same time the locations of the nodes of the Higgs field move towards the origin, as illustrated in Fig. 6 for very large values of the scalar coupling $\lambda$.

With help of Fig. [6 we can now address the limit $\lambda \rightarrow \infty$ for these new $\alpha$-branches. As $\lambda$ increases without bound, the minimal value $\alpha_{\min }$, beyond which the new branches exist, decreases towards zero. (Note, that $\alpha_{\min } \sim 1 / \sqrt{\lambda}$ for very large $\lambda$.) At the same time the scaled mass of the critical solutions at $\alpha_{\text {min }}$ decreases towards the mass of the generalized BM solution with $n=3$ [20], while their mass itself diverges. Likewise, the values of their metric functions at the origin approach those of the $n=3$ generalized BM solution [21]. Their nodes finally move continuously towards the origin, and the solutions shrink to zero size as $\lambda \rightarrow \infty$. Scaling the coordinates and Higgs field again reveals the generalized BM solution as limiting solution of finite scaled mass and finite scaled size [16, 17].

This limiting behavior for the critical solutions on the new $\alpha$-branches is similar to that observed for the solutions on the second $\alpha$-branches of the fundamental solutions. In both cases the respective generalized BM solution is approached.

One can understand the reason for this behaviour here, by noting that, as the scalar field becomes infinitely heavy, it decouples in these solutions and, in the double limit of infinite $\lambda$ and zero $\eta$, it does not affect the dynamics of the gauge sector anymore. In that sense the EYMH system is getting actually truncated here to an EYM system. 

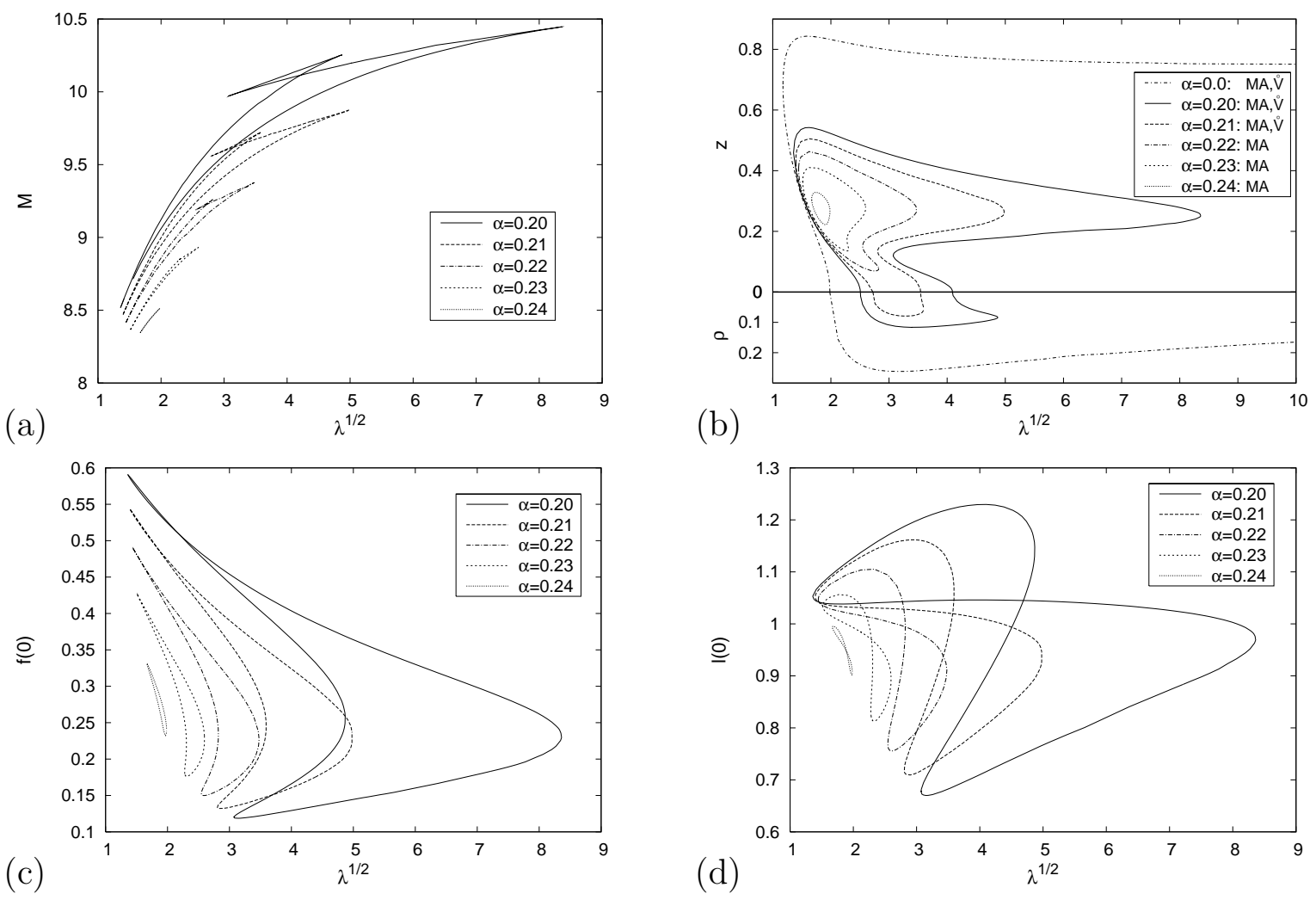

Figure 5: (a) The mass $M$ of $m=2, n=3$ solutions, related to the new flat-space $\lambda$-branches versus $\lambda$ for several values of the coupling $\alpha$. (b) The location of the isolated nodes $z$ and the radius $\rho$ of the vortex ring of the Higgs field, and the values of metric functions at the origin, $f(0)(\mathrm{c})$ and $l(0)(\mathrm{d})$ for the same set of solutions. (See Table 1 for the notation of the node and vortex ring configurations of the Higgs field.)

\subsection{Topologically nontrivial sector: $m=3, n=3$}

\subsubsection{Flat space solutions}

Let us now turn to solutions in the topologically nontrivial sector with $m=3, n=3$. In these solutions a triply charged monopole resides at the origin, providing the topological charge of the solutions. Again we first address the flat-space solutions, reviewing and supplementing previous results [10].

For $\lambda \rightarrow 0$, the $m=3, n=3$ solution possesses a triply charged monopole at the origin and two oppositely oriented vortex rings located symmetrically above and below the $x y$-plane [9]. For larger values of $\lambda$ one expects a bifurcation to occur, where new branches of solutions appear, which possess a different node structure, with three isolated nodes on the symmetry axis. For high values of $\lambda$ such a monopole-antimonopole chain (MAC) should then represent the energetically most favourable solution. 


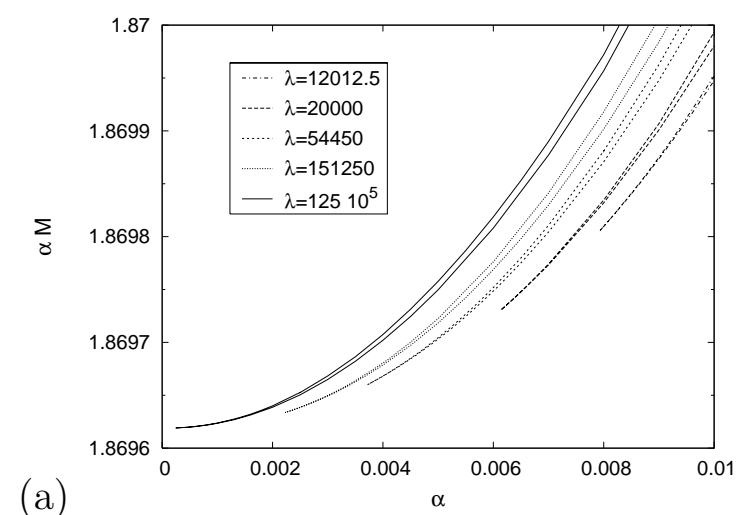

(a)

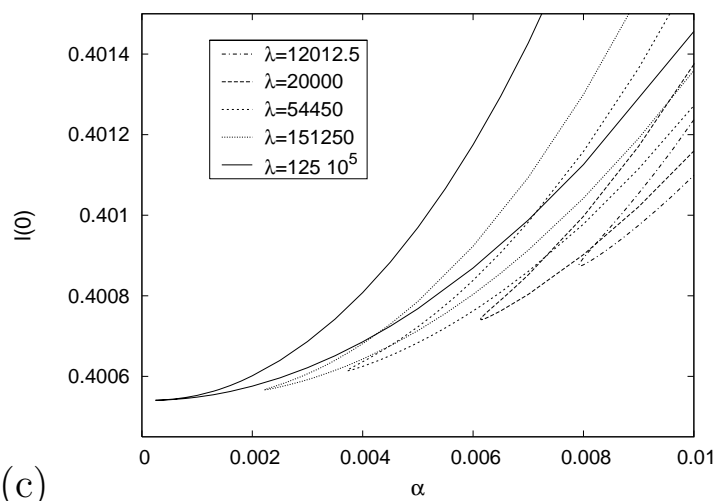

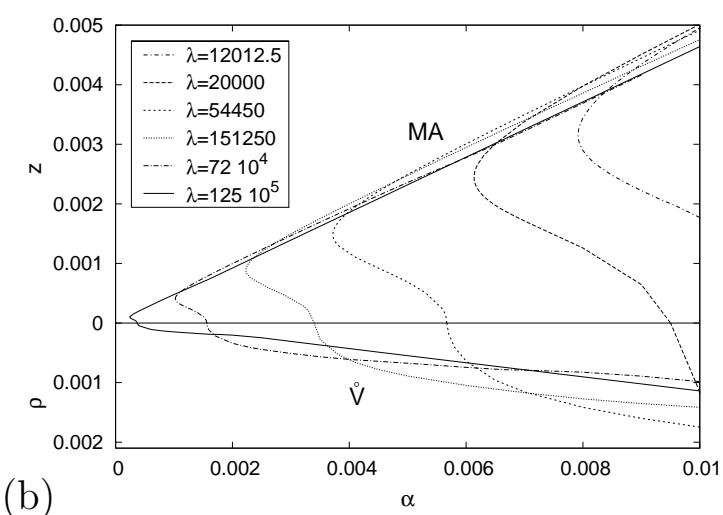

(b)

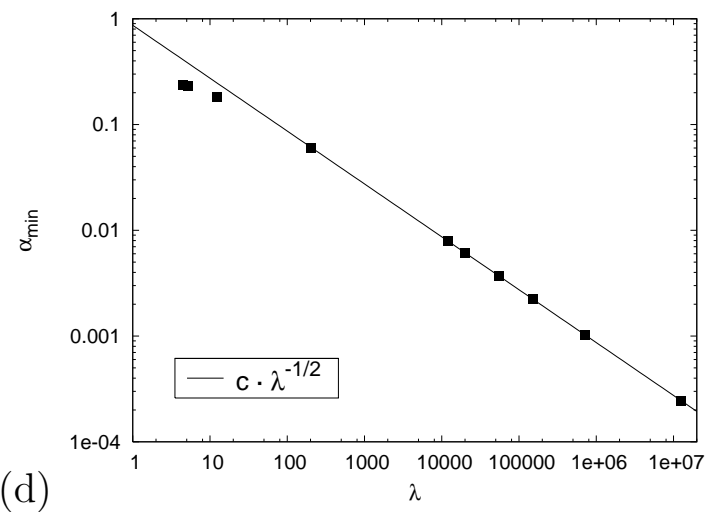

Figure 6: (a) The scaled mass $\alpha M$ of $m=2, n=3$ solutions, related to the new flat-space $\lambda$-branches versus $\alpha$ for very large values of the scalar coupling $\lambda$. (b) The location of the isolated nodes $z$ and the radius $\rho$ of the vortex ring of the Higgs field, the value of metric functions at the origin, $l(0)$ (c) for the same set of solutions. (d) The minimal value $\alpha_{\min }$ of the new $\alpha$-branches of $m=2, n=3$ solutions versus $\lambda$. (See Table 1 for the notation of the node and vortex ring configurations of the Higgs field.)

Indeed, at $\lambda_{c_{1}}=0.8099$ two new branches of solutions appear which possess the node structure of MACs [9]. But as seen in Fig. 7, the vortex ring solutions on the first (fundamental) $\lambda$-branch turn already into MACs before $\lambda_{c_{1}}$ is reached: As $\lambda$ increases, two nodes emerge from the origin and separate from each other along the $z$-axis. The solutions then possess three nodes on the symmetry axis and two vortex rings, located symmetrically above and below the $x y$-plane. As $\lambda$ increases further, the new nodes move further apart, while the vortex rings shrink to zero size, merging with the new nodes on the symmetry axis.

Thus beyond a certain value of $\lambda$, which is smaller than the first critical value $\lambda_{c_{1}}$, no vortex ring solutions are present any longer [9]. The fundamental branch and the new upper branch then merge at $\lambda_{c_{2}}=0.8194$, while the new lower branch continues to large values of $\lambda$, representing the fundamental branch there. This is seen in Fig. 7, where 

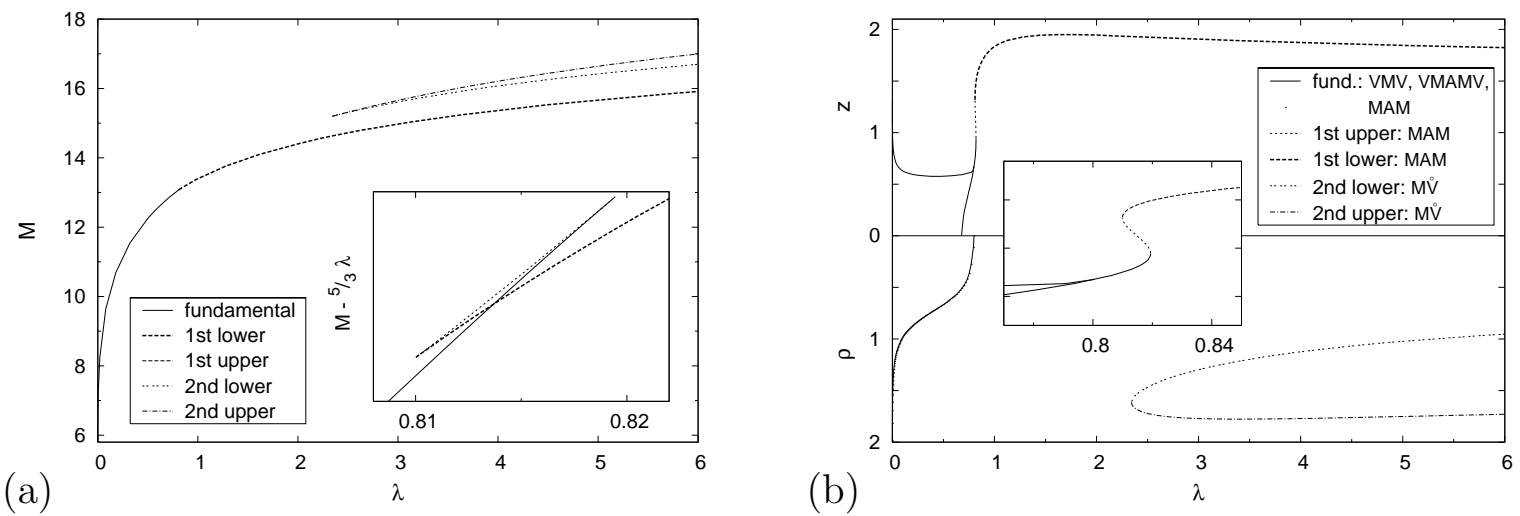

Figure 7: (a) The mass $M$ of the flat-space solutions on the fundamental $\lambda$-branch as well as on the additional $\lambda$-branches for $m=3, n=3$ solutions. (b) The location of the isolated nodes $z$ and vortex rings $(\rho, z)$ of the Higgs field for the same set of solutions. (See Table 2 for the notation of the node and vortex ring configurations of the Higgs field.)

the mass and the node structure of the $m=3, n=3 \lambda$-branches are exhibited, with emphasis on the three branches of MACs present in the small range of $\lambda, \lambda_{c_{1}} \leq \lambda \leq \lambda_{c_{2}}$.

Interestingly, a further bifurcation arises at $\lambda_{c_{3}}=2.3436$, which was missed before. At $\lambda_{c_{3}}$ two new branches of vortex ring solutions appear, whose single ring is located in the $x y$-plane. Thus beyond $\lambda_{c_{3}}$, vortex ring solutions are present again, but possess higher masses than the fundamental MAC solutions. These new $\lambda$-branches are also exhibited in Fig. 7 ,

\subsubsection{Gravitating solutions}

When gravity is coupled, branches of gravitating solutions arise from these $m=3$, $n=3$ flat-space configurations. Depending on the value of $\lambda$, two or more $\alpha$-branches are present, whose nature is considered in detail in the following.

The $\alpha$-dependence of the $\lambda<\lambda_{c_{1}}$ solutions is simple. A first $\alpha$-branch emerges from the flat-space solution and merges at some $\alpha_{\max }$ with a second $\alpha$-branch, which connects to the generalized BM solution in the limit $\alpha \rightarrow 0$. Indeed, for small values of $\alpha$ on the second branch, the solutions may be thought of as composed of a scaled $n=3$ generalized $\mathrm{BM}$ solution in an inner region and a flat-space charge-3 monopole configuration in an outer region [17]. In the limit $\alpha \rightarrow 0$, the mass of the solutions diverges, while their scaled mass approaches the mass of the generalized $n=3 \mathrm{BM}$ solution.

Solutions in the range $\lambda_{c_{1}} \leq \lambda \leq \lambda_{c_{2}}$

While for $\lambda<\lambda_{c_{1}}$ only a single flat-space solution is present, there are three flatspace solutions in the range $\lambda_{c_{1}} \leq \lambda \leq \lambda_{c_{2}}$. This complicates the picture considerably. We exhibit in Fig. 8 the node structure of the $\alpha$-branches, associated with all three flat 


\begin{tabular}{|c|c|}
\hline MAM & $\begin{array}{c}\text { A monopole-antimonopole chain with two monopoles } \\
\text { on the symmetry axis, one above and one below the } \\
x y \text {-plane, and an antimonopole at the origin. }\end{array}$ \\
\hline VMV & $\begin{array}{c}\text { A monopole at the origin and two vortex rings, one } \\
\text { above and one below the } x y \text {-plane. }\end{array}$ \\
\hline VMV monopole at the origin and a vortex ring in the \\
$x y$-plane.
\end{tabular}

Table 2: Configurations of the nodes and vortex rings of the Higgs field for $m=3, n=3$ solutions.

space solutions, for several values of $\lambda$ in this critical range.

While the left part of the figure shows the evolution of the zeros of the Higgs field with increasing scalar coupling $\lambda$, the right part gives for a particular value, $\lambda=0.815$, the assignment of the branches, to which the zeros belong. The $\alpha$-branch emerging from the fundamental $\lambda$-branch is labeled c, and the $\alpha$-branches emerging from the new lower $\lambda$-branch and the new upper $\lambda$-branch are labeled $\mathrm{a}$ and $\mathrm{b}$, respectively.

It is intuitively clear that in the complicated pattern of interaction between the monopoles and antimonopoles, the gravitational attraction plays a role similar to the scalar interaction, thus one can expect that coupling to gravity may provide a compensation for the weakening of the scalar interaction. This is indeed, what we here observe regarding the existence of the solutions and their node structure. For increasing $\alpha$, the branches a and b merge and disappear, while the branch c persists, but changes character, reversing the steps seen for the fundamental flat-space $\lambda$-branch in Fig. 7.

Indeed, at $\alpha=0$, all three branches begin as MAC solutions. As $\alpha$ increases, the two MAC branches a and b merge and end at a small $\alpha_{\max }$. The branch $\mathrm{c}$, however, extends 

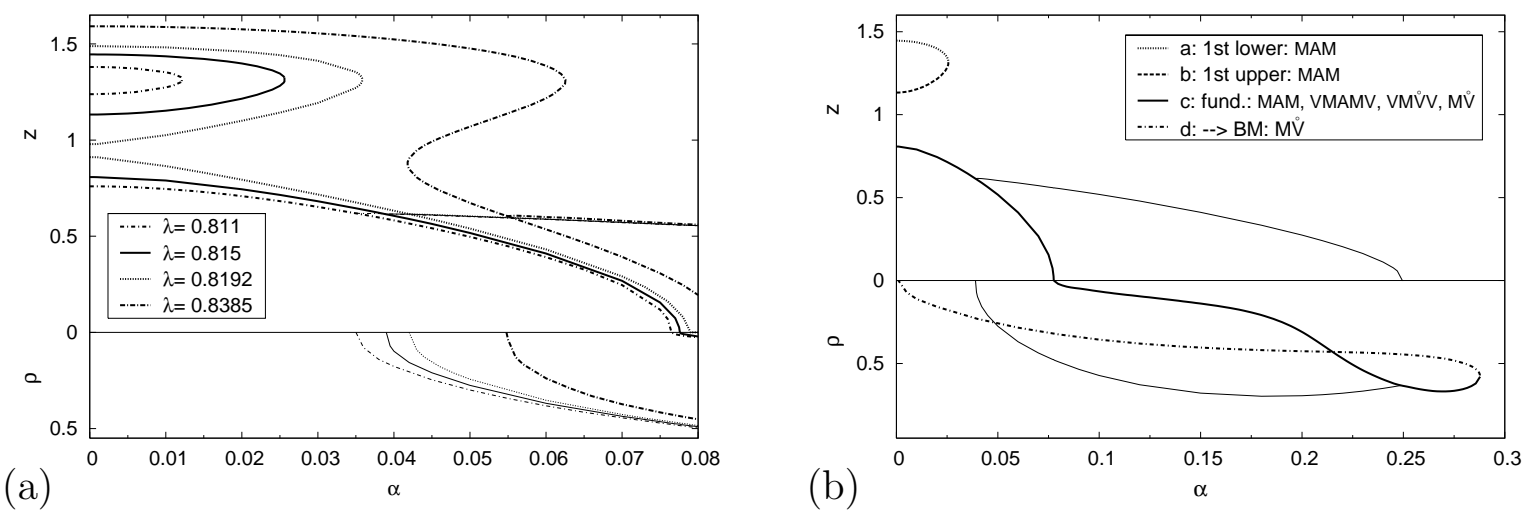

Figure 8: (a) The location of the isolated nodes $z$ and vortex rings $(\rho, z)$ of the Higgs field of $m=3, n=3$ solutions versus $\alpha$ for several values of the scalar coupling $\lambda$ in the critical range $\lambda_{c_{1}} \leq \lambda \leq \lambda_{c_{2}}$ and beyond. (b) The isolated nodes and vortex rings of the Higgs field for the complete set of solutions for $\lambda=0.815$. (See Table 2 for the notation of the node and vortex ring configurations of the Higgs field.)

further and changes its node structure accordingly, as $\alpha$ increases. First a vortex ring emerges from each outer node. Next the nodes move towards each other, merging at the origin. Then a central vortex ring appears in the $x y$-plane, and finally the outer rings merge with the central ring.

The branch c still persists to somewhat larger values of $\alpha$, and then merges at an $\alpha_{\max }$ with a second branch of vortex ring solutions labeled d. As expected, this branch extends backwards to $\alpha=0$, where it connects to a generalized BM solution.

The evolution of the solutions with increasing $\lambda$ can now be understood from the left part of the figure. As $\lambda$ increases, the branches a and $b$ extend to larger values of $\alpha$, before they merge. At the same time the nodes of the branches $b$ and $c$ approach each other. At the critical value $\lambda_{c_{2}}$ the two flat-space branches merge, and their nodes coincide. As $\lambda$ increases further, this critical point (where two branches merge) is retained, but it moves to finite values of $\alpha$. Note, that the pattern of nodes of the gravitating solutions in the interval [0.03 $<\alpha<0.07]$ for $\lambda=0.8385$ exhibited in Fig. 8, nicely reflects the (reversed) pattern of nodes of the flat-space solutions in the interval $[0.8<\lambda<0.85]$ exhibited in Fig. 7.

Emergence of the new flat-space branches at $\lambda_{c 3}$

As seen above, the bifurcations of the flat-space branches at $\lambda_{c_{1}}$ and $\lambda_{c_{2}}$ are reflected by bifurcations in curved space. In particular, the critical values of the gravitational coupling strength $\alpha$, where bifurcations arise for a given value of the scalar coupling strength $\lambda$, move towards smaller values of $\alpha$ with decreasing $\lambda$. As the minimal critical value of $\alpha$ reaches zero, the flat-space bifurcation point $\lambda_{c_{2}}$ arises.

It is thus obvious, that the new flat-space $\lambda$-branches arising at $\lambda_{c_{3}}$, should also have 

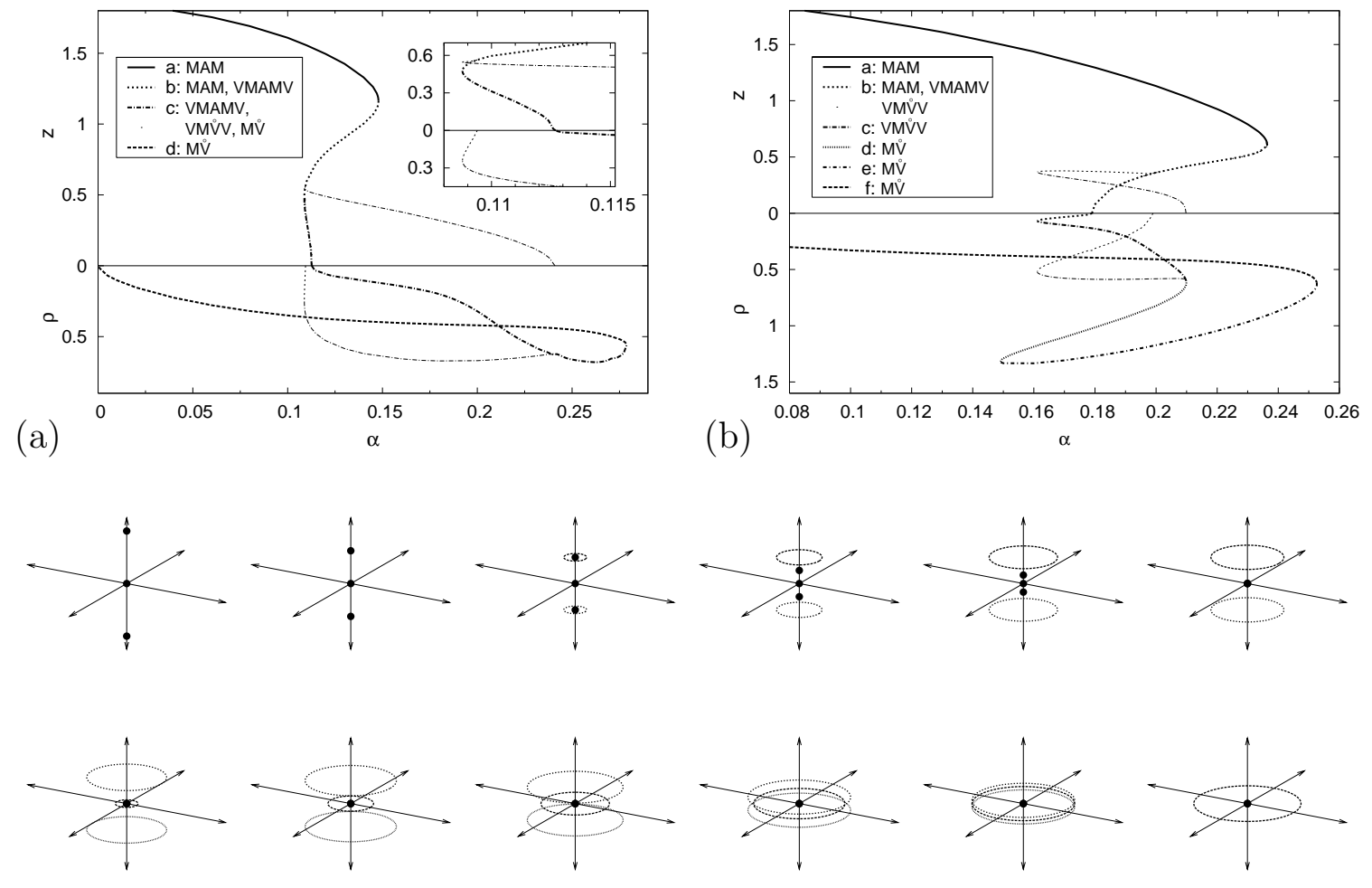

(c)
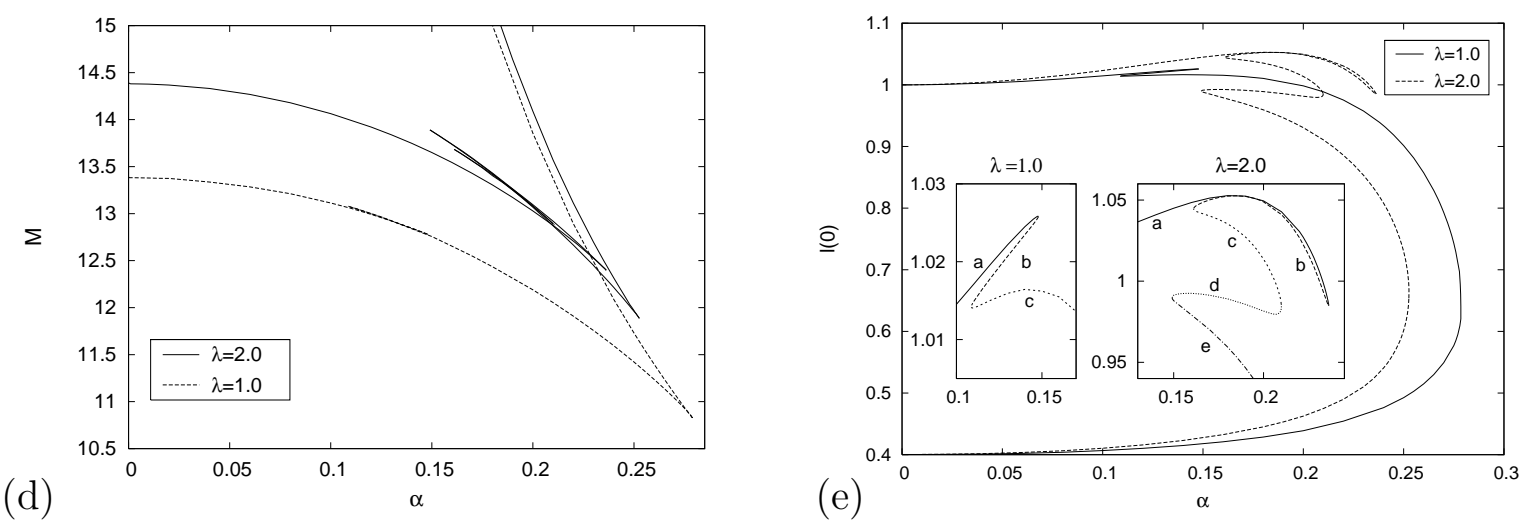

Figure 9: The location of the isolated nodes $z$ and vortex rings $(\rho, z)$ of the Higgs field for the complete set of solutions for $\lambda=1$ (a) and $\lambda=2$ (b) versus $\alpha$. Change of the structure of the nodes of the Higgs field following the evolution of the $\alpha$-branches (c). The mass $M(\mathrm{~d})$ and the value of metric function at the origin $l(0)$ (e) for the same set of solutions. 
precursors in curved space. These precursors indeed led to the detection of the new flat-space branches. In the following we analyze the branches of gravitating solutions, obtained with increasing scalar coupling strength $\lambda$, to clarify the emergence of the new flat-space branches.

Let us first consider the sets of solutions at $\lambda=1$ and $\lambda=2$, exhibited in Fig. 9, While the node structure nicely reveals the types of solutions present at the respective value of $\lambda$, and their evolution with $\alpha$, the values of the metric functions at the origin are instructive to get an overview of the sets of solutions present. For better identification, we again label the various branches of solutions a, b, c, etc.

For $\lambda=1$ there are four $\alpha$-branches, just like for the smaller $\lambda$ values of Fig. 8 . When moving continuously along these $\alpha$-branches, the node structure changes from MAC, present for $\mathrm{a}$ and most of $\mathrm{b}$, to MAC plus vortex rings, shortly before the bifurcation with c. The size and the location of the nodes and the rings then evolves along $\mathrm{c}$ until a single ring is left in the $x y$-plane (together with the always present node at the origin). This node structure is then retained on branch $d$, where the solutions evolve towards the generalized BM solution as $\alpha \rightarrow 0$.

As $\lambda$ increases further, interestingly, another bifurcation arises, and two more $\alpha$ branches appear. Consequently, there is now an interval of $\alpha$ with six $\alpha$-branches. The branches labeled a to $f$ are exhibited in the figure for $\lambda=2$. Moving continuously along the branches, one again observes the structure of the solutions change from MACs to MACs plus vortex rings, and then to a monopole plus vortex ring(s).

Most important for understanding the emergence of the new flat-space branches is the evolution of the branches $\mathrm{d}$ and $\mathrm{e}$ with $\lambda$, with particular emphasis on the critical value of $\alpha$, where these two branches merge. We therefore consider in Fig. 10 sets of solutions with still higher values of $\lambda$.

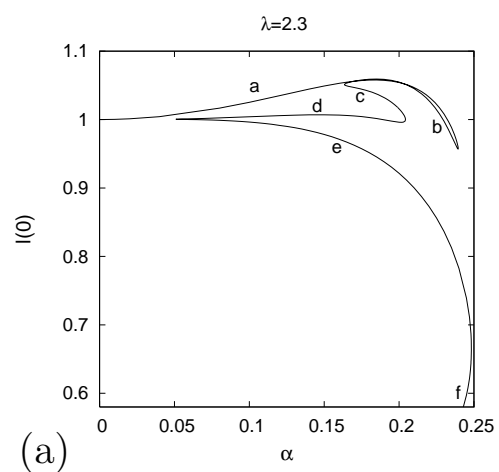

Figure 10: The value of metric function at the origin, $l(0)$, for $\lambda=2.3$ (a) and $\lambda=3.92$ (b) and for $\lambda=6.48$ (c).

As $\lambda$ increases further, the branches $\mathrm{d}$ and e extend to increasingly smaller values of $\alpha$. At the critical value $\lambda_{c_{3}}$ of the scalar coupling, the critical value of $\alpha$, where the 
branches $d$ and e merge, then precisely reaches zero, giving rise to the bifurcation point of the new flat-space solutions.

For values of the scalar coupling beyond $\lambda_{c_{3}}$, the set of solutions then splits into two disconnected parts. The first part emerges with branch a from the respective fundamental flat-space solution, evolves via branch b and branch c, and reaches along branch $d$ the new upper mass flat-space solution in the limit $\alpha \rightarrow 0$. The second part emerges with branch e from the new upper mass flat-space solution, and evolves with branch $f$ towards the limiting generalized BM solutions in the limit $\alpha \rightarrow 0$. This is demonstrated in Fig. 10 for the set of solutions with $\lambda=3.92$.

Solutions at large $\lambda$

In the case of the $m=2, n=3$ solutions we observed the appearance of new $\alpha$-branches of solutions for large values of $\lambda$, exhibiting an intriguing behaviour: In the limit $\lambda \rightarrow \infty$ the minimal value $\alpha_{\text {min }}$, beyond which these new branches exist, decreases towards zero, while the critical solutions themselves approach the generalized $\mathrm{BM}$ solution as $\alpha_{\min } \rightarrow \infty$.

We here observe an analogous pattern for the $m=3, n=3$ solutions. To see this pattern arising, we reconsider the evolution of the branches $a$ and $b$ with $\lambda$, exhibited in Fig. 9 and Fig. 10. As $\lambda$ increases, the branches $a$ and $b$ first elongate towards larger values of $\alpha$. Then at a critical value of $\lambda$, a new bifurcation arises, and two additional branches appear. The minimal value $\alpha_{\text {min }}$ possible for these new branches now decreases with increasing $\lambda$, analogous to the case of the respective branches of the $m=2, n=3$ solutions.

\subsection{Topologically trivial sector: $m=4, n=3$}

\subsubsection{Flat space solutions}

We now turn to the $m=4, n=3$ solutions, which reside in the topologically trivial sector. As before we first address the flat-space solutions, reviewing and supplementing previous results [10].

We exhibit in Fig. 11 the mass and the node structure of the $m=4, n=3$ flatspace $\lambda$-branches. The fundamental $\lambda$-branch is present for arbitrary scalar coupling strength. Its solutions possess two vortex rings, located symmetrically with respect to the $x y$-plane.

At $\lambda_{c_{1}}=0.491$ a first bifurcation arises, where a pair of new $\lambda$-branches appears, possessing higher mass and a mixed node structure, with two outer nodes on the symmetry axis and a vortex ring in the $x y$-plane [10]. With increasing $\lambda$ the solutions on the lower mass branch retain their node structure, while the upper mass branch solutions transform first into MAC solutions with four isolated nodes on the symmetry axis, and then back to the mixed node structure. 

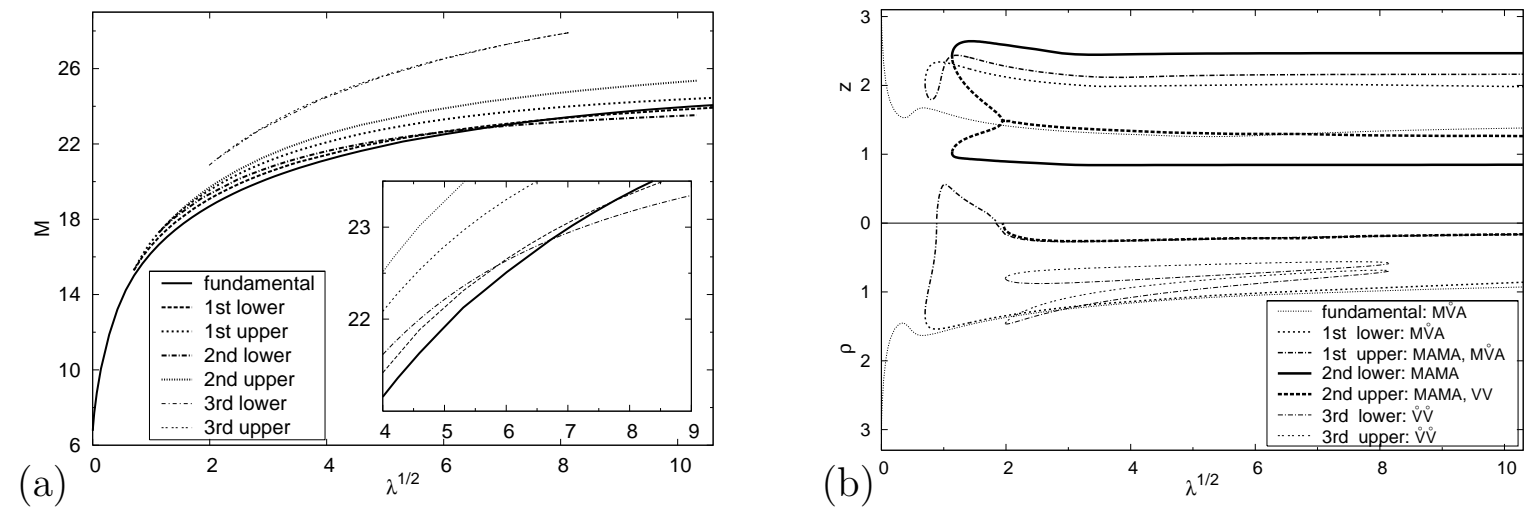

Figure 11: (a) The mass $M$ of the flat-space solutions on the fundamental $\lambda$-branch as well as on the additional $\lambda$-branches for $m=4, n=3$ solutions. (b) The location of the isolated nodes $z$ and vortex rings $(\rho, z)$ of the Higgs field for the same set of solutions. (See Table 3 for the notation of the node and vortex ring configurations of the Higgs field.)

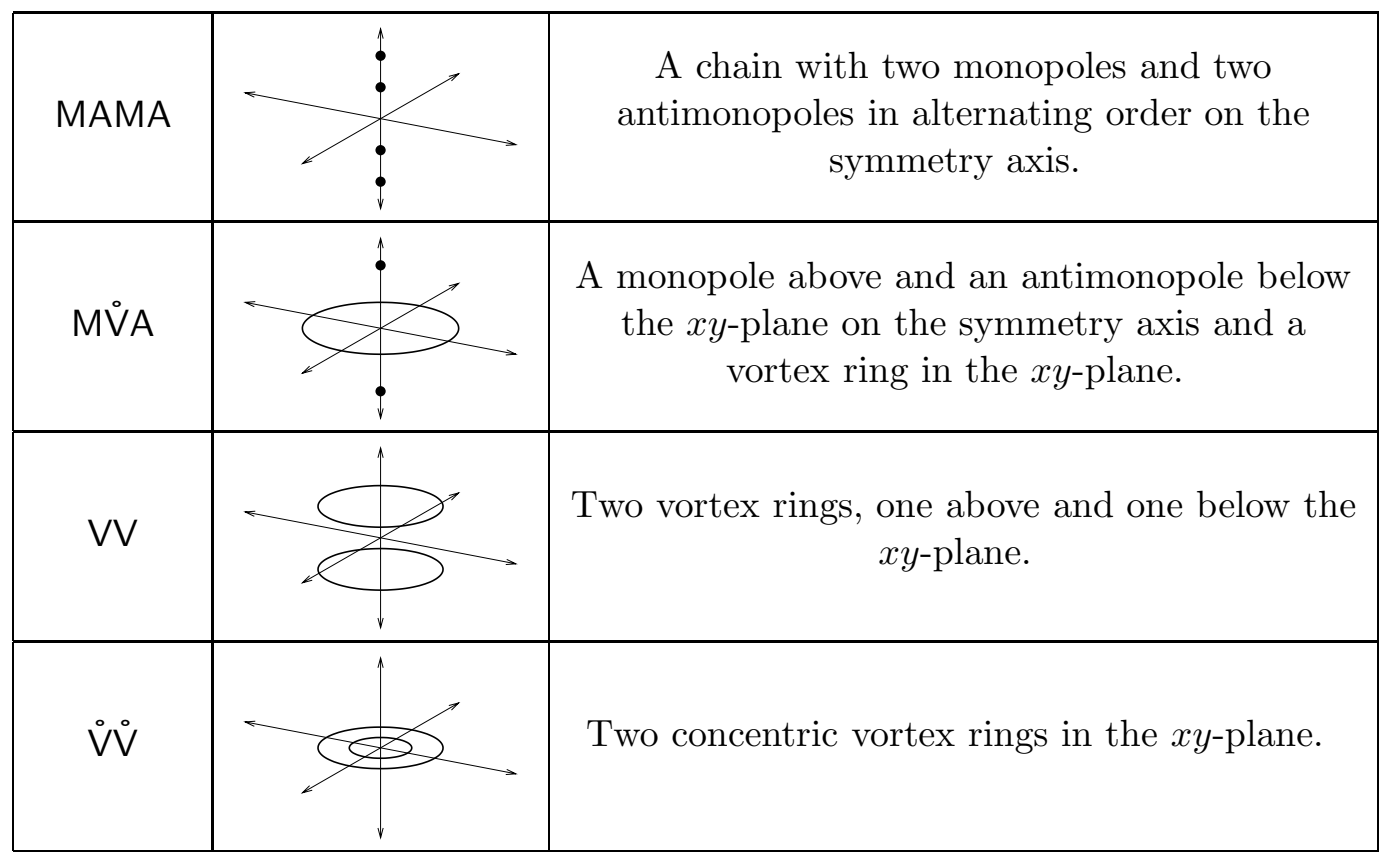

Table 3: Configurations of the nodes and vortex rings of the Higgs field for $m=4, n=3$ solutions.

Concerning the mass of these solutions, a transition between the fundamental branch and the new lower mass branch is observed at $\lambda_{m}^{1} \approx 59.8$, making the new lower branch solutions energetically favourable. As for the $m=2$ and $m=3$ solutions, it appears 
energetically advantageous for the $m=4$ solutions to exchange vortex rings for isolated nodes, when the scalar coupling is large.

We therefore argued before [10], that a further bifurcation was likely to exist, where another pair of branches would appear, representing MAC solutions with four isolated nodes on the symmetry axis. The solutions on this (conjectured) lower (mass) branch should then become the energetically most favourable configurations for large values of $\lambda$.

Indeed, as seen in Fig. 11, such a second bifurcation arises at $\lambda_{c_{2}}=1.288$, and a pair of MAC solutions appears. With increasing $\lambda$ the solutions on the lower mass branch retain the MAC node structure, while the node structure of the upper mass branch solutions changes and forms two vortex rings. Concerning the mass of the solutions we also observe the conjectured transition: beyond $\lambda_{m}^{2} \approx 45.2$ the new MAC branch has the lowest mass and thus becomes the energetically favoured branch.

Surprisingly, at $\lambda_{c_{3}}=3.962$ a third bifurcation arises and a third pair of branches appears with a new type of node structure. These solutions possess two concentric vortex rings in the $x y$-plane. Consequently their mass is considerably higher than the mass of the other configurations.

But these two branches of solutions have another interesting feature. Unlike the other branches of solutions, this third pair of branches exists only in a relatively small range of the scalar coupling. At the bifurcation point $\lambda_{c_{4}}=66.354$, the pair of branches merges and disappears again.

Since this third pair of branches of solutions appeared unexpectedly, the existence of further branches of flat-space solutions in certain ranges of the scalar coupling seems possible.

\subsubsection{Gravitating solutions}

When gravity is coupled, branches of gravitating solutions arise from all of these $m=4$, $n=3$ flat-space configurations. But as seen above, such $\alpha$-branches can bifurcate many times, leading to a plethora of gravitating solutions for larger values of $\lambda$. We therefore refrain from obtaining the complete picture for these $m=4, n=3$ solutions, and present only results for a few selected values of $\lambda$. In particular, we exhibit in Fig. 12 and Fig. 13 the mass, the node structure and the metric function value $l(0)$ versus $\alpha$, for sets of gravitating $m=4, n=3$ solutions with $\lambda=1,2$ and $\lambda=4.5$.

The first value $\lambda=1$ is chosen in the interval $\lambda_{c_{1}}<\lambda<\lambda_{c_{2}}$, after the first bifurcation. So there are three flat-space solutions, each giving rise to an $\alpha$-branch. The $\alpha$-branch emerging from the fundamental flat-space solution connects via a second $\alpha$-branch to the generalized BM solution, while the $\alpha$-branches emerging from the first new pair of flat-space solutions merge with each other. By considering the evolution of the nodes on the new upper mass branch with $\lambda$ and with $\alpha$, respectively, we note again, that an increase of the scalar coupling can lead to an analogous effect as the decrease of the 

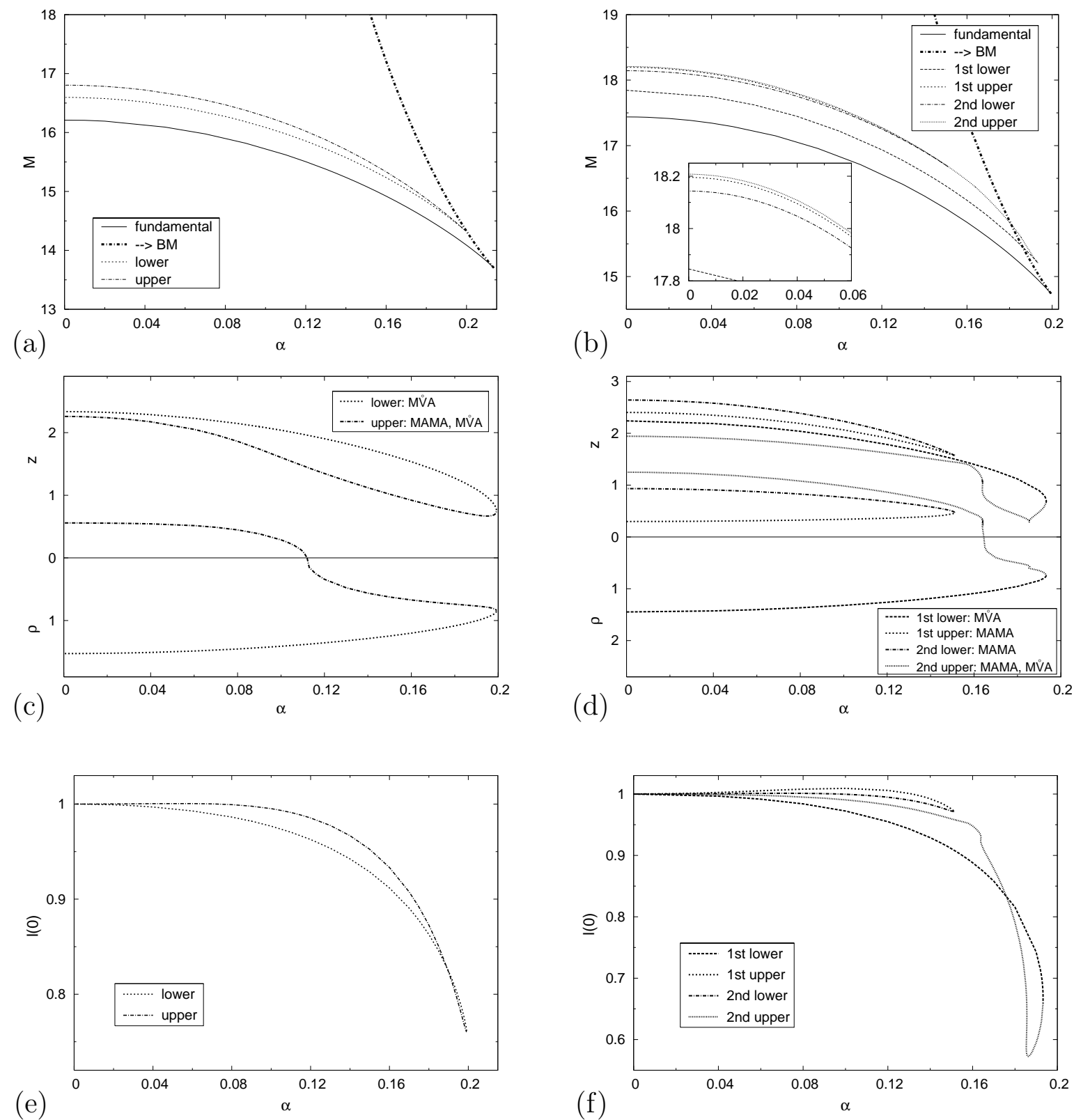

Figure 12: The mass $M$ of $m=4, n=3$ solutions versus $\alpha$ for $\lambda=1$ (a), $\lambda=2$ (b). The location of the isolated nodes $z$ and vortex rings $(\rho, z)$ of the Higgs field for the same set of solutions for $\lambda=1$ (c), $\lambda=2$ (d), and likewise the value of the metric function at the origin $l(0)$ for $\lambda=1$ (e), $\lambda=2$ (f). (See Table 3 for the notation of the node and vortex ring configurations of the Higgs field.) 
gravitational coupling. In both cases, the MAC node structure can change into a mixed node structure at a critical value of the coupling.

The second value $\lambda=2$ resides in the interval $\lambda_{c_{2}}<\lambda<\lambda_{c_{3}}$, after the second bifurcation. Thus there are five flat-space solutions, each giving rise to an $\alpha$-branch. As in the previous case, the $\alpha$-branch emerging from the fundamental flat-space solution connects via a second $\alpha$-branch to the generalized BM solution, and this feature of the fundamental branch solutions is retained, independent of $\lambda$.

However, the $\alpha$-branches emerging from the first new pair no longer merge with each other. Instead the first new lower branch merges with the second new upper branch, and the first new upper branch merges with the second new lower branch. The reason for this can be inferred from the evolution of the $\alpha$-branches with $\lambda$, since the emergence of the second new pair of flat-space solutions can be traced back to the appearance of a bifurcation on one of the $\alpha$-branches of the first new pair of solutions, for a value of $\lambda>1$. As $\lambda$ then increases, the two additional $\alpha$-branches grow in size, while their bifurcation point $\alpha_{\text {min }}$ moves towards smaller values of $\alpha$, reaching $\alpha=0$ at $\lambda_{c_{2}}$. Thus beyond $\lambda_{c_{2}}$, the $\alpha$-branches of the first pair of solutions are disconnected from each other and merge instead with those of the second pair.

As seen in the figure, at $\lambda=2$ the solutions on the $\alpha$-branch emerging from the first lower mass solution possess a mixed node structure, those connected to the second upper mass solution have a node structure changing from MAC to mixed, while the solutions on the other branches have MAC structure.

The third value of $\lambda$ is chosen in the interval $\lambda_{c_{3}}<\lambda<\lambda_{c_{4}}$, where three pairs of flat-space branches are present. Their corresponding $\alpha$-branches are exhibited in Fig. 13. At this value of $\lambda$ a plethora of $\alpha$-branches is present, caused by numerous further bifurcations. The node structures of these solutions range from MACs to double vortex rings. Thus one may speculate about the existence of solutions with still more complicated node structures, which might arise from new $\alpha$-branches, as the scalar coupling is further increased.

\section{Conclusions}

We have investigated static axially symmetric solutions of $S U(2)$ Einstein-Yang-MillsHiggs theory, representing monopole-antimonopole pairs, chains, vortex rings, and new types of configurations with mixed node structure. Such new configurations appear for larger values of the scalar coupling, when the subtle interplay between repulsive and attractive forces allows for more than one non-trivial equilibrium configuration of these systems.

In flat space, at critical values of the scalar coupling, bifurcations arise, where pairs of new branches of solutions appear, which possess a different node structure than the solutions on the fundamental branches. In particular, new solutions appear, where 

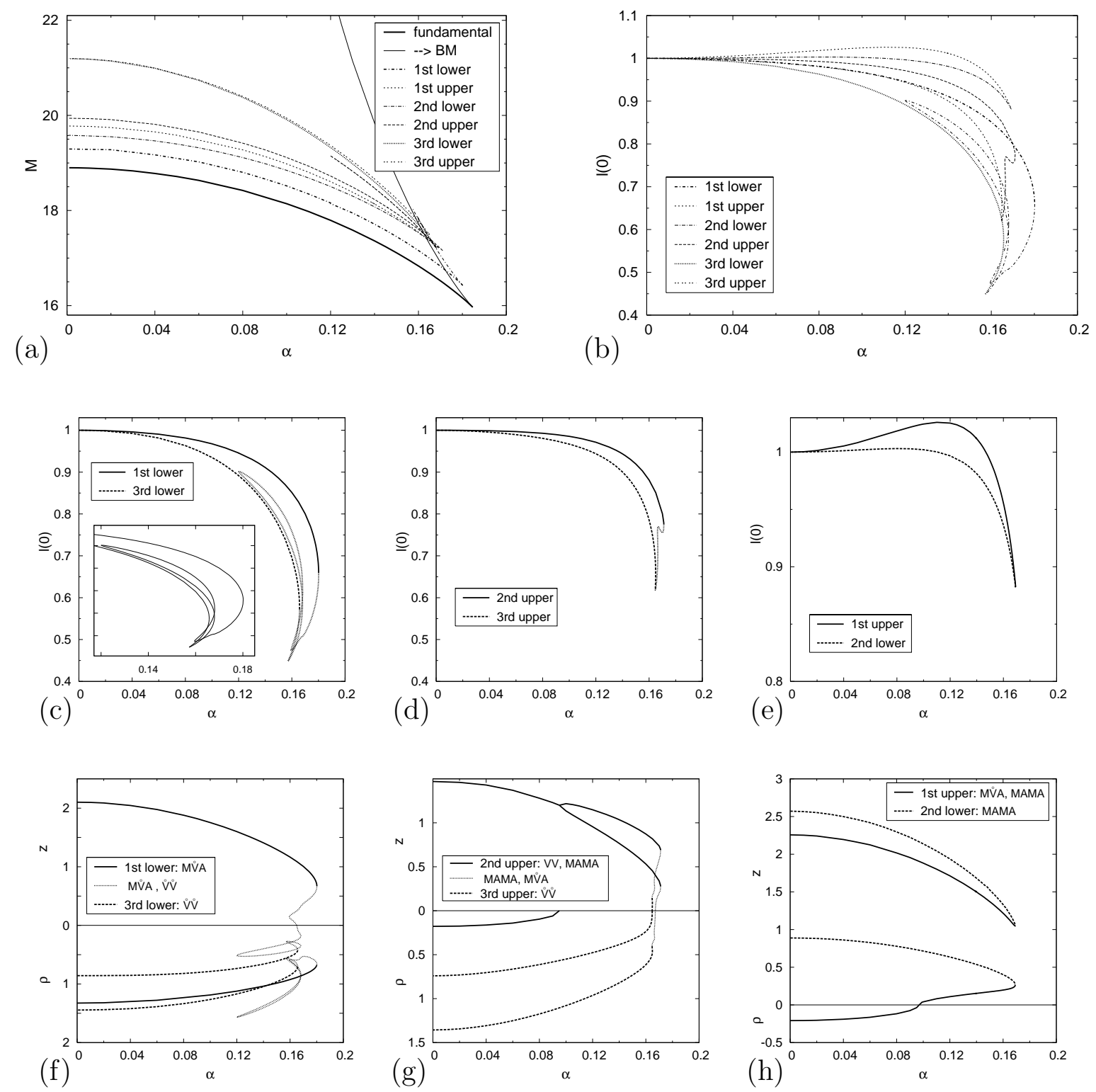

Figure 13: The mass $M$ of $m=4, n=3$ solutions versus $\alpha$ for $\lambda=4.5$ (a). The value of metric function at the origin, $l(0)$, for the complete set of solutions related to the new flat space $\lambda$-branches (b) and for the first (c), second (d) and third (e) pair of $\alpha$-branches emerging from the flat space $\lambda$-branches. The location of the isolated nodes $z$ and vortex rings $(\rho, z)$ of the Higgs field for the solutions emerging the first (d), second (e) and third (f) pair of $\alpha$-branches. (See Table 3 for the notation of the node and vortex ring configurations of the Higgs field.) 
vortex rings present in the fundamental solutions, are replaced by isolated nodes on the symmetry axis. For high values of $\lambda$ the new solutions with monopole-antimonopole chain structure have the lowest mass.

While we have studied here in detail only the monopole-antimonopole systems with $m=2,3,4$ and $n=3$, we conjecture, that this phenomenon is not restricted to these particular systems but that it is of a more general nature, implicating an enormous richness of configuration space for high values of $\lambda$ and larger $m$ and $n$. For $m=4$ and $n=3$, for instance, we observed already seven distinct flat-space solutions in a certain range of the scalar coupling.

The coupling to gravity leads to additional attraction between the components of these monopole-antimonopole systems, shifting the balance of forces and thus affecting the possible equilibrium configurations. Whereas for small scalar coupling only two gravitating branches of solutions are associated with a flat-space solution, there is a surge of further branches when the scalar coupling becomes large.

Particular of these branches can be shown to give rise to new pairs of flat-space solutions. Thus study of the Einstein-Yang-Mills-Higgs configuration space gives valuable insight into the configuration space of Yang-Mills-Higgs theory. Indeed, by exploiting this interrelationship, we were able to find the new flat-space solutions presented.

But the Einstein-Yang-Mills-Higgs configurations are also related to Einstein-YangMills solutions. First of all, in the limit $\alpha \rightarrow 0$, one of the $\alpha$-branches of a particular set of solutions, characterized by $m, n$ and $\lambda$, always connects to a generalized BartnikMcKinnon solution [19, 20, 21]. But another connection arises in the limit of infinite scalar coupling. In this limit the bifurcation point $\alpha_{\min }$ of two of the $\alpha$-branches is seen to approach $\alpha=0$, while the solutions at $\alpha_{\min }$ approach the respective generalized Bartnik-McKinnon solution.

\section{Acknowledgement}

We would like to acknowledge valuable discussions with Burkhard Kleihaus, Eugen Radu, Tigran Tchrakian, and Michael Volkov. Ya.S. would like to thank the Department of Mathematical Physics, NUI Maynooth, and the SPhT CEA/Saclay, for their kind hospitality.

\section{References}

[1] G. 't Hooft, Nucl. Phys. B79 (1974) 276;

A. M. Polyakov, Pis'ma JETP 20 (1974) 430.

[2] E.J. Weinberg, and A.H. Guth, Phys. Rev. D14 (1976) 1660.

[3] C. Rebbi, and P. Rossi, Phys. Rev. D22 (1980) 2010. 
[4] R.S. Ward, Comm. Math. Phys. 79 (1981) 317;

P. Forgacs, Z. Horvath, and L. Palla, Phys. Lett. 99B (1981) 232;

M.K. Prasad, Comm. Math. Phys. 80 (1981) 137;

M.K. Prasad, and P. Rossi, Phys. Rev. D24 (1981) 2182.

[5] B. Kleihaus, J. Kunz, and D. H. Tchrakian, Mod. Phys. Lett. A13 (1998) 2523.

[6] C. H. Taubes, Commun. Math. Phys. 86 (1982) 257;

C. H. Taubes, Commun. Math. Phys. 86 (1982) 299;

C. H. Taubes, Commun. Math. Phys. 97 (1985) 473.

[7] W. Nahm, unpublished;

Bernhard Rüber, Diploma Thesis, University of Bonn, 1985.

[8] B. Kleihaus, and J. Kunz, Phys. Rev. D61 (2000) 025003.

[9] B. Kleihaus, J. Kunz, and Ya. Shnir, Phys. Lett. B570 (2003) 237;

B. Kleihaus, J. Kunz, and Ya. Shnir, Phys. Rev. D68 (2003) 101701;

B. Kleihaus, J. Kunz, and Ya. Shnir, Phys. Rev. D70 (2004) 065010.

[10] J. Kunz, U. Neeman and Ya. Shnir, Phys. Lett. B640 (2006) 57.

[11] N. J. Hitchin, N. S. Manton and M. K. Murray, Nonlinearity 8 (1995) 661;

C. J. Houghton and P. M. Sutcliffe, Commun. Math. Phys. 180 (1996)343;

C. J. Houghton and P. M. Sutcliffe, Nonlinearity 9 (1996) 385;

P. M. Sutcliffe, Int. J. Mod. Phys. A12 (1997) 4663.

[12] N. S. Manton, Nucl. Phys. B126 (1977) 525;

W. Nahm, Phys. Lett. 85B (1979) 373;

N. S. Manton, Phys. Lett. 154B (1985) 397.

[13] B. Hartmann, B. Kleihaus, and J. Kunz, Phys. Rev. Lett. 86 (2001) 1422;

B. Hartmann, B. Kleihaus, and J. Kunz, Phys. Rev. D65 (2001) 024027.

[14] B. Kleihaus, J. Kunz and U. Neemann, Phys. Lett. B 623 (2005) 171.

[15] K. Lee, V.P. Nair, and E.J. Weinberg, Phys. Rev. D45 (1992) 2751;

P. Breitenlohner, P. Forgacs, and D. Maison, Nucl. Phys. B383 (1992) 357;

P. Breitenlohner, P. Forgacs, and D. Maison, Nucl. Phys. B442 (1995) 126.

[16] B. Kleihaus, and J. Kunz, Phys. Rev. Lett. 85 (2000) 2430.

[17] B. Kleihaus, J. Kunz, and Ya. Shnir, Phys. Rev. D71 (2005) 024013. 
[18] A. Lue and E.J. Weinberg, Phys. Rev. D60 (1999) 084025;

P. Breitenlohner, P. Forgacs, and D. Maison, in preparation.

[19] R. Bartnik, and J. McKinnon, Phys. Rev. Lett. 61 (1988) 141.

[20] B. Kleihaus and J. Kunz, Phys. Rev. Lett. 78 (1997) 2527;

B. Kleihaus and J. Kunz, Phys. Rev. D57 (1998) 834.

[21] R. Ibadov, B. Kleihaus, J. Kunz, and Ya. Shnir, Phys. Lett. B609 (2005) 150.

[22] Y. Brihaye, and J. Kunz, Phys. Rev. D50 (1994) 4175.

[23] W. Schönauer, and R. Weiß, J. Comput. Appl. Math. 27 (1989) 279;

M. Schauder, R. Weiß, and W. Schönauer, The CADSOL Program Package, Universität Karlsruhe, Interner Bericht Nr. 46/92 (1992). 